
Pathophysiology of Haemostasis and Thrombosis

Received: J anuary 28, 2002

Accepted in revised form: J une 28, 2002

\title{
Abnormally Short Activated Partial Thromboplastin Times Are Related to Elevated Plasma Levels of TAT, F1+2, D-Dimer and FVIII:C
}

\author{
Edwin ten Boekel Piet Bartels \\ Laboratory for Clinical Chemistry, Hematology and Immunology, Medical Center of Alkmaar, \\ Alkmaar, The Netherlands
}

\section{Key Words}

Short APTT - Thrombin-antithrombin III complex . Prothrombin fragment F1+2 · Factor VIII

\begin{abstract}
Abnormally short activated partial thromboplastin times (APTTs) are associated with an increased risk of thrombotic disorders. We have examined the status of coagulation activity in subjects with short APTTs. In addition, the presence of the thrombotic risk factors G1691A-factor V, G20210A-prothrombin gene mutation and factor VIII coagulant activity (FVIII:C) was determined. Plasma levels of TAT, F1+2, D-dimer and FVIII:C were markedly higher in subjects with short APTTs compared with subjects with normal APTTs. APTTs were inversely related to TAT, F1+2, D-dimer and FVIII:C levels. The prevalence of G1691A-factor V and G20210A-prothrombin gene mutation between the group with short APTTs and the control group was not significantly different. Hence, these gene polymorphisms do not contribute to the increased risk of thrombosis associated with short APTTs. In conclusion, short APTTs are indicative of marked coagulation activity and elevated FVIII:C levels. Elevated FVIII:C levels may play a pathogenic role in the increased risk of thrombosis associated with abnormally short APTTs.
\end{abstract}

Copyright (c)2002 S. Karger AG, Basel

\section{Introduction}

Thrombotic disorders are one of the most common causes of morbidity and mortality in the Western world. The pathophysiologic basis of hypercoagulability is complex and multifactorial. Several genetic factors are implicated in the pathogenesis of venous thrombosis [1]. The G1691A mutation in factor $\mathrm{V}$ gene, also known as factor V Leiden (FVL), and the prothrombin G20210A gene mutation are the most commonly found hereditary defects that contribute to venous thromboembolism $[2,3]$. In addition, increased levels of plasma factor VIII coagulant activity (FVIII:C) have been shown to be associated with thrombosis [4-7].

The activated partial thromboplastin time (APTT) assay is generally used to assess coagulation disorders in patients with abnormal bleeding due to deficiencies within the intrinsic coagulation cascade. Inherited coagulopathies tend to induce prolonged APTTs. The clinical significance of prolonged APTTs is well known, whereas that of short APTTs has not been fully explored.

Several studies have indicated that a short APTT may be considered as a risk factor for hypercoagulability. One prospective clinical study found that a short APTT is related to a 10-fold increased incidence of thromboembolic events [8]. A recent prospective study demonstrated

\footnotetext{
Dr. E. ten Boekel

Laboratory for Clinical Chemistry, Hematology and Immunology

Medical Center of Alkmaar, PO Box 501

NL-1800 AM Alkmaar (The Netherlands)

Tel. +31 72548 3613, Fax +31 72548 2175, E-Mail E.ten.Boekel@mca.nl
} 
Table 1. Numbers and percentages of patients per hospital department

\begin{tabular}{lrrrrr}
\hline Department & \multicolumn{2}{c}{ APTT $<26 \mathrm{~s}$} & & \multicolumn{2}{c}{ APTT 28-38 s } \\
\cline { 2 - 3 } \cline { 5 - 6 } & $\mathrm{n}$ & $\%$ & & $\mathrm{n}$ & $\%$ \\
\hline First aid & 91 & 45 & & 50 & 50 \\
Cardiology & 22 & 11 & & 8 & 8 \\
Intensive care unit & 40 & 20 & & 21 & 21 \\
Neurology & 8 & 4 & & 2 & 2 \\
Internal medicine & 18 & 9 & & 14 & 14 \\
Oncology & 2 & 1 & & 0 & 0 \\
Others & 21 & 10 & & 5 & 5 \\
\hline
\end{tabular}

that patients with short APTTs are at increased risk for thromboembolism, in particular venous thrombosis (odds ratio: 5.4) [9]. Reddy et al. [10] reported that approximately $25 \%$ of patients with short APTTs will develop thrombotic events within 1 year after detection, whereas in the control group the incidence was less than $3 \%$. An increased risk of thrombosis in patients with short APTTs was observed in several other studies [11-15]. Lowe et al. [16] concluded following multivariate analyses that out of 29 studied hemostatic parameters, only a shorter APTT and activated protein $C$ resistance are associated with an increased risk of postoperative deep vein thrombosis.

Since short APTTs are common laboratory findings, a growing demand in our hospital is noticed for the elucidation of the clinical impact of a short APTT. Moreover, the underlying mechanism of increased thrombotic risk has not been examined yet. In order to explore the pathogenesis of thrombosis related to short APTTs, we evaluated the status of coagulation activity and determined the prevalence of two common genetic risk factors for thrombotic diseases.

\section{Materials and Methods}

Experimental Design, Subjects and Blood Sampling

The study included patients hospitalized in the Medical Center Alkmaar. During a period of 9 months, all specimens sent to our laboratory for APTT requests were included. Blood was sampled from an antecubital vein by venipuncture and anticoagulated with $0.129 M$ sodium citrate (Becton Dickinson Vacutainer, Meylan, France). After centrifugation at $1,550 \mathrm{~g}$, APTT was determined in plasma within $1 \mathrm{~h}$ after blood sampling. Aliquots of plasma and blood cells were immediately frozen and stored at $-70^{\circ} \mathrm{C}$ until further testing. A total number of 202 consecutive subjects with APTT $<26$ s and 100 consecutive subjects with a normal APTTs (control group) were tested. Of each subject, only one APTT was included.
Determination of APTT and Level of FVIII:C

APTT was determined using the ILTest ${ }^{\text {TM }}$ APTT-SP (synthetic phospholipid/silica) kit on an ACL 3000 analyser (Instrumentation Laboratory, Milano, Italy) according to the manufacturer's protocol. The reference range of APTT in apparently healthy subjects is 28 38 s. FVIII:C was measured by the conventional one-stage clotting assay performed on an Instrumentation Laboratory (IL) ACL analyser using IL APTT-lyophilized Silica reagent and factor-VIII-deficient plasma (Organon Teknika, Cambridge, UK). The reference range of FVIII:C in apparently healthy subjects is $70-150 \%$.

\section{Assay of TAT, F1+2, D-Dimer and C-Reactive Protein}

Levels of thrombin-antithrombin III (TAT) complex and prothrombin fragment $\mathrm{F} 1+2$ in plasma were determined using an enzyme immunoassay (Enzygnost, Dade Behring, Marburg, Germany) according to the manufacturer's recommendations. Reference ranges of healthy subjects: TAT $<4.1 \mu \mathrm{g} / 1 ; \mathrm{F} 1+2<1.1 \mathrm{nmol} / \mathrm{l}$. Plasma concentration of D-dimer was established by an enzyme-linked immunosorbent assay performed on a Vidas analyser (bioMérieux, Marcy-l'Etoile, France) according to the manufacturer's instructions. Reference range D-dimer $<400 \mu \mathrm{g} / \mathrm{l}$. C-reactive protein (CRP) was measured with a turbidimetric method on a Synchron CX system (Beckman Coulter Inc., Fullerton, Calif., USA). The reference range of CRP is $<3.0 \mathrm{mg} / \mathrm{l}$.

\section{Determination of Factor V-G1691A (FVL) and}

Prothrombin-G20210A Genotype

Genomic DNA was isolated from peripheral blood leukocytes using a DNA isolation kit for mammalian blood (Roche Diagnostics Ltd., Basel, Switzerland) according to the manufacturer's instructions. FVL genotype was determined by allele specific restriction enzyme analysis as described before [2]. G20210A-prothrombin genotyping was performed as originally reported by Poort et al. [3].

\section{Statistical Analysis}

Student's 2-tailed t test was used to determine the significance of differences between groups of subjects with different genotypes. A p value $<0.05$ was considered to be statistically significant.

\section{Results}

A total number of 10,450 blood samples were tested for APTT. Abnormally short APTTs $(<26 \mathrm{~s})$ were detected in 256 samples $(2.4 \%)$ which were derived from 202 different patients. Only the blood sample obtained from the first blood collection of each of the 202 patients was tested further. The prevalence of short APTTs is similar to that reported elsewhere $[9,10]$. Hence, a short APTT is a frequent laboratory observation. The majority of patients with short APTTs were admitted to the First Aid Department and Intensive Care Unit (table 1). Similar results were found for the control group consisting of patients with APTTs within the reference range (table 1). The diagnosis and medical history of the studied subjects were not evaluated since this was beyond the aim of the present 
study. Though, we have examined the prevalence of thrombotic diseases in 31 patients with short APTTs and 34 patients with normal APTTs consecutively admitted to the First Aid Department. Thrombotic disease, which concerned an acute myocardial infarction or stroke in all cases, was established in $6(19 \%)$ patients with short APTTs and $6(18 \%)$ patients with normal APTTs. These results indicate that the prevalence of thrombotic disease in the patient group with short- and normal APTTs is similar. In support, short APTTs have not been related to specific diagnoses [9].

In order to examine whether short APTTs are caused by ex vivo artifacts, we have confirmed the short APTT on repeated testing in samples of 30 consecutive patients obtained from repeated blood collection. The repeated APTT was determined within $5 \mathrm{~h}$ after the initial blood collection. A repeated short APTT was detected in 28 patients. Only 2 patients showed APTTs $>26$ s for the second blood sample. Both of these patients had APTTs of $25 \mathrm{~s}$ in the first collected blood sample. We conclude that short APTTs are unlikely caused by ex vivo activation of coagulation due to preanalytic artifacts.

\section{Levels of TAT, F1+2 and D-Dimer}

Plasma samples of subjects with APTT $<26 \mathrm{~s}$ were tested for TAT, F1+2 and D-dimer levels. Results are shown in figure 1 and table 2. Plasma TAT, F1+2 and D-dimer levels in patients with APTTs $<26 \mathrm{~s}$ were found to be significantly higher than those in the control group [mean differences $(95 \% \mathrm{CI})$ : TAT $=23.2(18.0-27.0) \mu \mathrm{g} / \mathrm{l}$, $\mathrm{p}<0.0001 ; \mathrm{F} 1+2=2.3(1.7-2.9) \mathrm{nmol} / \mathrm{l}, \mathrm{p}<0.0001$; and $\mathrm{D}$-dimer $=1,662(1,290-2,034) \mu \mathrm{g} / 1, \mathrm{p}<0.0001]$. APTTs were inversely related to TAT, F1+2 and D-dimer levels (fig. 1). If the patients are grouped according to their APTTs, then all patients with APTTs $<26$ s showed significantly increased plasma TAT $(\mathrm{p}<0.0001), \mathrm{F} 1+2(\mathrm{p}<$ $0.0001)$ and D-dimer $(\mathrm{p}<0.0001)$ levels compared with the control group. Hence, significantly elevated TAT, $\mathrm{F} 1+2$ and D-dimer levels were already found in the patient group with APTTs $=25 \mathrm{~s}$ compared with the control group [mean differences $(95 \% \mathrm{CI})$ : TAT $=12.6(6.7-$ 18.5) $\mu \mathrm{g} / \mathrm{l}, \mathrm{p}<0.0001 ; \mathrm{F} 1+2=1.7(0.8-2.6) \mathrm{nmol} / \mathrm{l}, \mathrm{p}<$ 0.0001 , and D-dimer $=1,228(714-1,741) \mu \mathrm{g} / \mathrm{l}, \mathrm{p}<$ $0.0001]$. APTTs $\leq 22 \mathrm{~s}$ coincided with markedly high levels of TAT (median value $>60 \mu \mathrm{g} / \mathrm{l}$ ) and $\mathrm{F} 1+2$ (median value $>6 \mathrm{nmol} / \mathrm{l})$, whereas subjects with APTTs $\geq 23 \mathrm{~s}$ showed median TAT and F1+2 levels of $<20 \mu \mathrm{g} / 1$ and $<3 \mathrm{nmol} / \mathrm{l}$, respectively (fig. 1).

Short APTT Is Associated with Coagulation

Activation
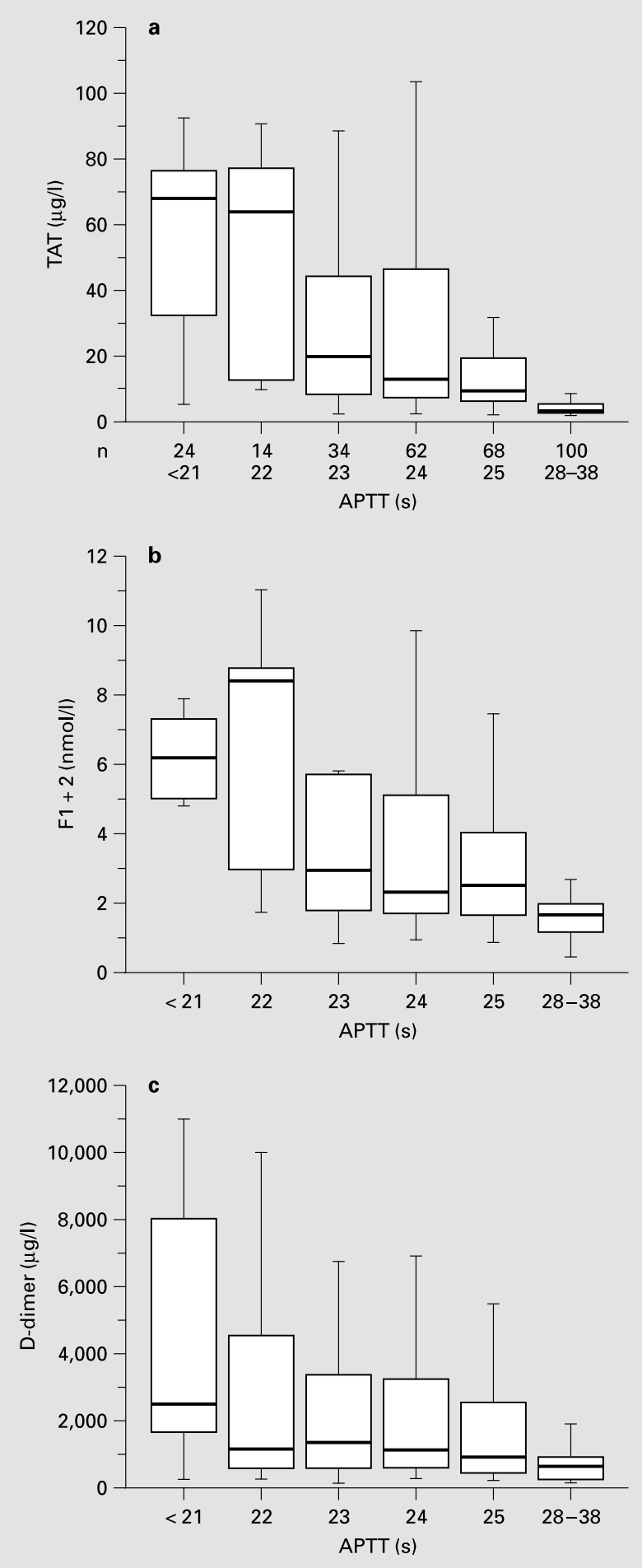

Fig. 1. Box and whisker plot of TAT (a), F1+2 (b) and D-dimer (c) levels in subjects with different APTTs. Numbers (n) of tested subjects are indicated. The lower and upper limits of each box represent the 25th and 75th percentile, respectively. The horizontal line in the box shows the median. The bars indicate the lowest and the highest observed values that were $<1.5$ times the box height away from the 25 th or 75 th percentile, respectively.

Pathophysiol Haemost Thromb 2002;32:137-142 


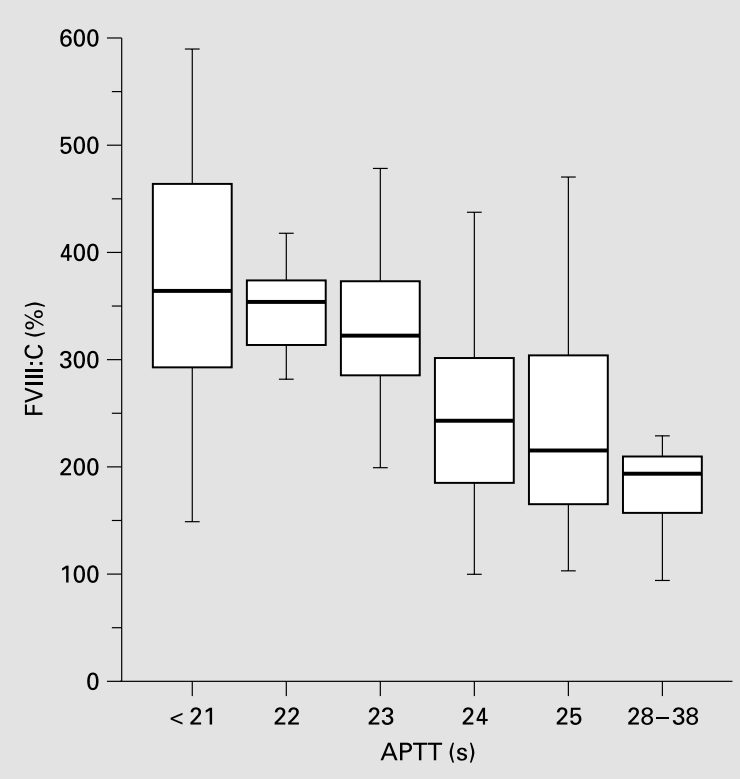

Fig. 2. Box and whisker plot of FVIII:C levels in subjects with different APTT values. The lower and upper limits of each box represent the 25th and 75th percentile, respectively. The horizontal line in the box shows the median. The bars indicate the lowest and the highest observed values that were $<1.5$ times the box height away from the 25 th or 75 th percentile, respectively. Numbers of tested subjects are the same as indicated in figure 1.

Table 2. Mean levels and $95 \% \mathrm{CI}$ of D-dimer, TAT, F1+2 products and FVIII:C

\begin{tabular}{lccccc}
\hline & \multicolumn{2}{c}{ APTT $<26 \mathrm{~s}$} & & \multicolumn{2}{c}{ APTT 28-38 s } \\
\cline { 2 - 3 } \cline { 6 - 7 } & mean & $95 \% \mathrm{CI}$ & & mean & $95 \% \mathrm{CI}$ \\
\hline D-dimer, $\mu \mathrm{g} / 1$ & 2,424 & $2,095-2,753$ & & 762 & $584-938$ \\
TAT, $\mu \mathrm{g} / \mathrm{l}$ & 28.8 & $25.0-32.7$ & & 5.6 & $3.2-8.0$ \\
F1+2, nmol/1 & 4.1 & $3.5-4.7$ & & 1.8 & $1.6-2.2$ \\
FVIII:C, $\%$ & 298 & $277-319$ & & 197 & $171-223$ \\
\hline
\end{tabular}

\section{Level of FVIII:C}

Increased levels of FVIII:C were detected in virtually all (93\%) patients with APTTs $<26 \mathrm{~s}$ (fig. 2). The level of FVIII:C is demonstrated to be gradually increased with decreasing APTT (fig. 2). FVIII:C levels in the groups of patients with APTT $<26$ s were significantly higher compared with the control group [mean difference $(95 \% \mathrm{CI})$ : 101 (74-128) \%, p < 0.0001] (table 2). FVIII:C levels were already significantly elevated in patients with APTTs $=$ $25 \mathrm{~s}$ [mean difference (95\% CI): 54 (14-94) $\%, \mathrm{p}=0.008$ ] compared with the control group.

In order to answer the question whether the increased FVIII levels are due to acute-phase reactions, plasma CRP levels were determined in 30 consecutive patients with short- and normal APTTs. Elevated CRP levels were established in 23\% (7/30) of the patients with APTTs $<26 \mathrm{~s}$ and in $40 \%(12 / 30)$ of patients with APTTs of 28 $38 \mathrm{~s}$. No relationship was found between CRP and the length of APTT (results not shown). These results indicate that acute-phase reactions are not a major determinant of the elevated FVIII:C levels associated with short APTTs.

\section{Prevalence of Genetic Risk Factors for Thrombosis}

We examined whether FVL and prothrombin G20210A polymorphisms contribute to the pathogenesis of increased thrombotic risk related to short APTTs.

The FVL mutation was detected in $10(5 \%)$ individuals of the group with APTTs $<26 \mathrm{~s}$. All 10 individuals were heterozygous for the FVL mutation. This aberration was not significantly different from the prevalence of $6 \%$ established in the control group. For the prothrombin mutation, $3(1.5 \%)$ subjects of group APTT $<26 \mathrm{~s}$ were detected to be heterozygotes and no subjects were homozygotes. Two percent were found to be heterozygous for this mutation in the control group. Thus, no significant difference in the prevalence of the prothrombin G20210A gene mutation was observed between subjects with short APTTs and controls.

\section{Discussion}

Several studies revealed an association between a short APTT and an increased risk of thromboembolism [8-15]. In the present study, we evaluated the coagulation status of patients with short APTTs. We found no evidence that carriership of factor V-G1691A or prothrombinG20210A gene mutation contributes to a shortening of the APTT and therefore to the increased risk of thrombosis associated with a short APTT.

Increased levels of TAT, F1+2, D-dimer and FVIII:C were detected in subjects with APTT $<26$ s. Elevated levels of TAT and F1+2 are indicative of increased thrombin generation which arises in plasma following activation of the coagulation system [17]. Hence, our results indicate that short APTTs are associated with activated coagulation. Enhanced plasma levels of F1+2 and FVIII in patients with short APTTs were also reported by others 
[9, 13, 18]. However, the relationship between APTTs and the level of F1+2 and FVIII:C has not been studied previously. We found that the levels of TAT, F1+2 and FVIII:C were inversely correlated with the APTTs. Our observations may imply that an increased FVIII:C shortens the APTT. Mansveldt et al. [19] reported that elevated FVIII:C levels are associated with relatively shorter APTTs although they remain within the reference range of healthy subjects. Hence, FVIII:C may not be the sole determinant inducing a short APTT.

A recent study has reported that the vast majority of subjects with venous thromboembolism with elevated FVIII:C levels show markedly increased TAT and F1+2 levels [20]. The authors hypothesized that an increased FVIII:C level exerts prothrombotic effects by increasing thrombin generation. Indeed, a weak correlation between thrombin generation and FVIII:C levels is established $[20,21]$. In this regard, a shortening of the APTT may result from accumulation of circulating activated coagulation factors in plasma caused by enhanced coagulation activation in vivo. Interestingly, short APTTs have been described in a significant number of patients with lowgrade disseminated intravascular coagulation, supporting the above statement [22]. Our data demonstrate that patients with short APTTs show substantial activation of the coagulation and fibrinolytic system.

The majority $(>90 \%)$ of the patients will have normal APTTs if measured several weeks or months later [8, unpubl obs.]. We hypothesize that short APTTs reflect an imbalanced coagulation system resulting in an increased thrombotic tendency. However, the perturbation of hemostasis itself may not be sufficient to induce short
APTTs. The coexistence of a second factor (e.g. caused by a disease) may be needed to trigger a short APTT revealing the patient's imbalanced coagulation system. The underlying mechanism of the increased risk of thrombotic events associated with short APTT has not been studied previously. Elevated levels of TAT, F1+2 and D-dimer are not necessarily indicative of an acute thromboembolic event and may not be predictive of future thrombosis [23]. We found that factor V-G1691A and prothrombinG20210A gene mutations in the patient group are present at frequencies similar to those in the control population. Further, the observed frequencies are in agreement with data obtained from studies on healthy individuals in earlier reports [24]. Hence, these genetic polymorphisms do not explain the thrombotic tendency of patients with short APTTs.

Increased FVIII:C activity has been shown to be an independent risk factor for thrombosis in several studies [4-7]. Factor VIII levels are increased in the acute-phase response, during pregnancy and oral contraception, conditions known to be associated with increased thromboembolic disease. In our studied population, only a very low percentage of subjects was pregnant and the majority revealed normal serum levels of CRP (an acute-phase reactant) suggesting that these factors may not be significant determinants of the increased levels of FVIII:C in subjects with short APTTs. In support of this O'Donnell et al. [5] reported that elevated levels of FVIII:C in subjects with venous thromboembolic disease are not associated with increased CRP levels. Elevated levels of FVIII:C may play a pathogenic role in the increased risk of thrombosis associated with abnormally short APTTs.

\section{References}

1 Lane DA, Grant PJ: Role of hemostatic gene polymorphisms in venous and arterial thrombotic disease. Blood 2000;95:1517-1531.

2 Bertina RM, Koeleman RPC, Koster T, Rosendaal FR, Dirven RJ, de Ronde H, van der Velden PA: Reitsma PH: Mutation in blood coagulation factor $\mathrm{V}$ associated with resistance to activated protein C. Nature 1994;369:64-67.

3 Poort SR, Rosendaal FR, Reitsma PH, Bertina RM: A common genetic variation in the $3^{\prime}$ untranslated region of the prothrombin gene is associated with elevated plasma prothrombin levels and an increase in venous thrombosis. Blood 1996;88:3698-3703.
4 Koster T, Blann AD, Briet E, Vandenbroucke JP, Rosendaal FR: Role of clotting factor VIII in effect of von Willebrand factor on occurrence of deep-vein thrombosis. Lancet 1995; 345:152-155.

5 O'Donnell J, Tuddenham EG, Manning R, Kemball-Cook G, Johnson D, Laffan M: High prevalence of elevated factor VIII levels in patients referred for thrombophilia screening: Role of increased synthesis and relationship to acute phase reaction. Thromb Haemost 1997; 77:825-828.

6 Kraaijenhagen RA, in 't Anker PS, Koopman MM, Reitsma PH, Prins MH, van den Ende A, Buller HR: High plasma concentration of factor VIII:C is a major risk factor for venous thromboembolism. Thromb Haemost 2000;83: 5-9.
7 Kyrle PA, Minar E, Hirschl M, Bialonczyk C, Stain M, Schneider B, Weltermann A, Speiser W, Lechner K, Eichinger S: High plasma levels of factor VIII and the risk of recurrent venous thromboembolism. N Engl J Med 2000;343: 457-462.

8 McKenna R, Bachmann F, Miro-Quesada M: Thromboembolism in patients with abnormally short activated thromboplastin time. Thromb Haemost 1977;38:893-899.

9 Korte W, Clarke S, Lefkowitz JB: Short activated partial thromboplastin times are related to increased thrombin generation and an increased risk for thromboembolism. Am J Clin Pathol 2000;113:123-127. 
10 Reddy NM, Hall SW, MacKintosh FR: Partial thromboplastin time. Prediction of adverse events and poor prognosis by low abnormal values. Arch Intern Med 1999;159:27062710.

11 Gallus AS, Hirsh J, Gent M: Relevance of preoperative and postoperative blood tests to postoperative leg-vein thrombosis. Lancet 1973;2: 805-809.

12 Pilgeram LO: Abnormalities in clotting and thrombolysis as a risk factor for stroke. Thromb Diath Haemorrh 1974;31:245-264.

13 Salem HH, Whitworth JA, Koutts J, KincaidSmith PS, Firkin BG: Hypercoagulation in glomerulonephritis. Br Med J (Clin Res Ed) 1981; 282:2083-2085.

14 Landi G, D'Angelo A, Boccardi E, et al: Venous thromboembolism in acute stroke: Prognostic importance of hypercoagulability. Arch Neurol 1992;49:279-283.

15 Guirguis N, Budisavljevic MN, Self S, Rajagopalan PR, Lazarchick J: Acute renal artery and vein thrombosis after renal transplant, associated with a short partial thromboplastin time and factor V Leiden mutation. Ann Clin Lab Sci 2000;30:75-78.
16 Lowe GD, Haverkate F, Thompson SG, Turner RM, Bertina PM, Turpie AG, Mannucci PM: Prediction of deep vein thrombosis after elective hip replacement surgery by preoperative clinical and haemostatic variables: The ECAT DVT study. European Concerted Action on Thrombosis. Thromb Haemost 1999; 81-879-886.

17 Bauer KA, Rosenberg RD: The pathophysiology of the prethrombotic state in humans: Insights gained from studies using markers of hemostatic system activation. Blood 1987;70: 343-350.

18 Humphries JE, Acker MN, Schnell DE, Knarr JW: Artifactual prolongation of the activated partial thromboplastin time mimicking a coagulopathy. Am J Clin Pathol 1993;100:108110.

19 Mansveldt EP, Laffan M, McVey JH, Tuddenham EG: Analysis of the F8 gene in individuals with high plasma factor VIII:C levels and associated venous thrombosis. Thromb Haemost 1998;80:561-565.
20 O’Donnell J, Mumford AD, Manning RA, Laffan MA: Marked elevation of thrombin generation in patients with elevated FVIII:C and venous thromboembolism. Br J Haematol 2001; 115:687-691.

21 Lowe GD, Rumley A, Woodward M, Morrison CE, Philippou H, Lane DA, Tunstall-Pedoe $\mathrm{H}$ : Epidemiology of coagulation factors, inhibitors and activation markers: The Third Glasgow MONICA Survey. Illustrative reference ranges by age, sex and hormone use. Br J Haematol 1997;97:775-784.

22 Bick RL: Disseminated intravascular coagulation: Pathophysiological mechanisms and manifestations. Semin Thromb Hemost 1998; 24:3-18.

23 Kyrle PA, Eichinger S, Pabinger I, Stumpflen A, Hirschl M, Bialonczyk C, Schneider B, Mannhalter C, Melichart M, Traxler G, Weltermann A, Speiser W, Lechner K: Prothrombin fragment $\mathrm{F} 1+2$ is not predictive for recurrent venous thromboembolism. Thromb Haemost 1997;77:829-833.

24 Bertina RM: The prothrombin $20210 \mathrm{G}$ to A variation and thrombosis. Curr Opin Hematol 1998;5:339-342. 